\section{The additional value of blood culture bottles in the diagnosis of endophthalmitis}

HS Tan", EAE Ghyczy-Carlborg1', L Spanjaard²

and MD de Smet ${ }^{1,3}$

\begin{abstract}
Purpose To assess the additional value of blood culture bottles (BCBs) in the diagnosis of endophthalmitis by comparing its culture yield with that of conventional media (CM). Design Retrospective consecutive case series. Methods We included patients who were treated between January 2001 and January 2010 for clinically suspected endophthalmitis of any etiology, and had vitreous specimens cultivated in both BCB and CM.

Results Specimens from 85 eyes from 85 patients were included. The culture yield of BCB was $69 \%$, and that of CM was $72 \%$ (difference not significant). Adding the results of BCB improved the yield of CM significantly by $13 \%$, resulting in a combined yield of $81 \%$. The sensitivity of detection of Haemophilus influenzae in BCB seemed lower compared with $\mathrm{CM}$, possibly due to the lack of growth factors in the BCB. There was no difference in yield between specimens obtained by tap or by vitrectomy.

Conclusion In contrast with earlier reports, we did not find $B C B$ superior to $C M$. The combined use of BCB and CM increased the pathogen detection rate significantly and should therefore be considered as the microbiological method of choice in the work-up of endophthalmitis.
\end{abstract}

Eye (2011) 25, 1069-1073; doi:10.1038/eye.2011.142; published online 17 June 2011

Keywords: endophthalmitis; blood culture bottles; Haemophilus influenzae

\section{Introduction}

Infectious endophthalmitis is a sightthreatening disease. Inoculation of an infectious agent into the eye can occur during or after ophthalmic surgery, as a result of penetrating trauma, or by dissemination through the blood stream. There are distinct patterns of microbiological etiologies that are linked to each clinical setting. ${ }^{1}$ In an acute postoperative period, the causative agent is usually a resident of skin, the eyelid margins, or tear-film of the patient. In the case of post-traumatic endophthalmitis, organisms are generally derived from the environment. Endogenous endophthalmitis usually occurs in debilitated or immunocompromised patients and is caused by a different spectrum of pathogens related to the underlying systemic disease. Prompt institution of appropriate antimicrobial treatment is crucial in the management of endophthalmitis. An accurate and timely identification of the infectious agent and confirming the appropriateness of the initial choice of antimicrobial therapy is essential to ensure a positive outcome to this often devastating disease. In the context of endogenous endophthalmitis, identification of the causative pathogen in the eye can orient treatment to an unsuspected systemic infection, and identify its cause.

Culture yields from vitreous fluid using agar-based conventional media (CM) are notoriously variable. Analogous to vitreous fluid, culture of other normally sterile body fluids, such as synovial, cerebrospinal, pleural, and peritoneal fluid, on CM is not always successful. The additional use of blood culture bottles (BCBs) has been shown to increase the culture yield of these fluids significantly. ${ }^{2}$ BCBs were found to enhance recovery of clinically significant isolates from these specimens, especially for fastidious and slow-growing microorganisms. ${ }^{2,3}$

The potential use of BCB for vitreous specimens has been the subject of recent publications. Using BCB seems to be a good
${ }^{1}$ Department of Ophthalmology, Academic Medical Center, University of Amsterdam, Amsterdam, The Netherlands

${ }^{2}$ Department of Medical Microbiology and Center for Infection and Immunity Amsterdam, Academic Medical Center, University of Amsterdam, Amsterdam, The Netherlands

${ }^{3}$ Retina and ocular inflammation unit, Center for Specialized Ophthalmology, Montchoisi Clinic, Lausanne,

Switzerland

Correspondence: HS Tan, Department of Ophthalmology, Academic Medical Center, University of Amsterdam, PO Box 22660, Amsterdam 1100 DD, The Netherlands Tel: + 3120566 4551; Fax: + 31842200663 . E-mail: h.tan@ amc.uva.nl

Received: 23 November 2010

Accepted: 12 April 2011 Published online: 17 June 2011 
alternative to $\mathrm{CM}$ in clinics without adequate laboratory facilities for microbiology $y^{4}$ and these studies reported yields that were higher than previously reported for CM. ${ }^{4,5}$ Direct comparison between the two techniques has been described in small series that suggest superior results for $\mathrm{BCB}$ compared with $\mathrm{CM} .^{6,7}$ The purpose of our current study was to determine the yields from BCB and $\mathrm{CM}$ in a larger series, in a setting with a good access to a microbiology laboratory.

\section{Materials and methods}

Medical records of consecutive patients treated for a clinically suspected endophthalmitis of any etiology between January 2001 and January 2010 were reviewed. The laboratory notes of obtained specimens in these cases were reviewed and we included those cases where a vitreous specimen was cultivated in parallel in both $\mathrm{BCB}$ and CM. We excluded cases where antibiotic treatment was instituted before vitreous sampling. All cases were treated at the Academic Medical Center Amsterdam, the Netherlands, a tertiary referral center. This study adhered to the tenets of the Declaration of Helsinki and was approved by the University of Amsterdam Institutional Review Board.

Undiluted vitreous specimens were obtained either via vitreous tap by aspiration through a 23-gauge needle or via mechanized vitreous biopsy through a sclerotomy at the start of a vitrectomy procedure. We did not systematically note sample size in each individual case, but typically, a tap would produce $0.3-0.5 \mathrm{ml}$ of undiluted vitreous, whereas a biopsy with a vitrectome would harvest $0.5-1 \mathrm{ml}$. At the end of the tap or vitrectomy procedure, the vitreous specimen was divided into three equal samples: one sample for $\mathrm{CM}$ was kept at $4^{\circ} \mathrm{C}$ and inoculated within $18 \mathrm{~h}$ on blood and chocolate agar $\left(37^{\circ} \mathrm{C}\right.$ in $\left.7 \% \mathrm{CO}_{2}\right)$ and on blood agar $\left(37^{\circ} \mathrm{C}\right.$ in anaerobic environment). The two other samples were immediately inoculated in the BCB. We used the BacT/Alert FAN aerobic and FAN anaerobic bottles (BioMérieux, Marcy l'Etoile, France). The bottles were incubated in the BacT/Alert instrument. All media were incubated during 7 days (in cases of chronic or possibly fungal endophthalmitis for 14 days) and processed according to standard microbiological practice.

The primary outcome measure was culture yield from BCB and CM. Parameters retrieved were presenting visual acuity (VA), cause of endophthalmitis (etiology) and method of obtaining the specimen (tap vs vitrectomy).

Statistical analysis was performed using SPSS software for Windows version 16.0 (SPSS Inc., Chicago, IL, USA) for the McNemar's $\chi^{2}$-test and the Pearson $\chi^{2}$-test. The
Institutional Review Board at the University of Amsterdam declared that this type of retrospective study waived the need for IRB approval, in accordance with Dutch law on human clinical trials. The Institutional review board at the University of Amsterdam declared that this type of retrospective study waived the need for IRB approval, in accordance with Dutch law on human clinical trials.

\section{Results}

Specimens from 85 eyes from 85 patients were included. From these specimens, BCB yielded $69 \%$ growth while $\mathrm{CM}$ yielded $72 \%$ growth. The difference in yield between the two systems was not statistically significant ( $P=0.815$; McNemar's $\chi^{2}$-test $)$. There were 16 specimens with negative growth in both systems, 47 specimens yielded matching pathogens in both systems, in 10 specimens a pathogen was found only in the CM system, and in 8 specimens the BCB system was the only to yield a pathogen. In the remaining four specimens, growth of different pathogens was found in the two systems. Polymicrobial growth was found in two BCB and two CM cultures.

Adding the results of $\mathrm{BCB}$ improved the yield of $\mathrm{CM}$ by $13 \%$, resulting a combined yield of $81 \%$. This improvement was statistically significant $(P<0.001$; McNemar's $\chi^{2}$-test).

In four cases (three post-injection and one postphacoemulsification), the suspicion for endophthalmitis was relatively low and negative culture results supported a final diagnosis of sterile endophthalmitis. In three cases of blebitis, cultures were negative. We did not exclude these cases from our series.

Gram stains of the vitreous samples were prepared and evaluated in 79 cases. In only 30 cases (38\%), the Gram stain identified the presence of microorganisms. Specimens with a positive Gram stain all had positive cultures. Moreover, staining and morphological characteristics of pathogens identified by Gram staining matched the culture result in all cases.

Culture results for the different etiologies are shown in Table 1. Endophthalmitis following phacoemulsification represented the largest group and showed the highest yield for both culture systems. Specimens from endogenous endophthalmitis had the lowest yield.

Table 2 depicts the spectrum of pathogens. Coagulasenegative staphylococci were encountered most often, followed by Streptococcus species. Gram-negative isolates were found in $15 \%$ of cases. Haemophilus influenzae was recovered in solid media in four cases, but BCB failed to show growth of this pathogen in three out of these 
Table 1 Endophthalmitis: culture results and etiology

\begin{tabular}{lrrr}
\hline & $\begin{array}{r}B C B, \\
\mathrm{n}(\%)\end{array}$ & $\begin{array}{c}C M, \\
\mathrm{n}(\%)\end{array}$ & $\begin{array}{c}\text { Combined, } \\
\mathrm{n}(\%)\end{array}$ \\
\hline Phacoemulsification $(n=51)$ & $38(75)$ & $39(77)$ & $45(88)$ \\
Trabeculectomy $(n=9)$ & $7(78)$ & $6(67)$ & $7(78)$ \\
Injection $(n=8)$ & $5(63)$ & $6(75)$ & $6(75)$ \\
Endogenous $(n=12)$ & $4(33)$ & $5(42)$ & $8(67)$ \\
Else $(n=5)^{\mathrm{a}}$ & $2(40)$ & $2(40)$ & $3(60)$ \\
Total $(n=85)$ & $59(69)$ & $61(72)$ & $69(81)$
\end{tabular}

Abbreviations: $\mathrm{BCB}$, blood culture bottles; $\mathrm{CM}$, conventional media. aElse: 2 trauma, 2 corneal ulcers, and 1 after strabismus operation.

Table 2 Endophthalmitis: number of positive cultures for different pathogens

\begin{tabular}{lrrr}
\hline & $B C B$ & $C M$ & Comb \\
\hline Coagulase-negative staphylococci & 25 & 26 & 30 \\
Staphylococcus aureus & 4 & 4 & 5 \\
Streptococcus species & 15 & 16 & 17 \\
Enterococcus species & 5 & 5 & 5 \\
Other Gram-positive bacteria & 4 & 4 & 4 \\
Haemophilus influenzae & 1 & 4 & 4 \\
Other Gram-negative bacteria & 6 & 4 & 7
\end{tabular}

Abbreviations: $\mathrm{BCB}$, blood culture bottles; $\mathrm{CM}$, conventional media; comb, combined.

Table 3 Endophthalmitis: positive culture in relation to presenting visual acuity

\begin{tabular}{lc}
\hline Presenting VA & $\mathrm{n}(\%)$ \\
\hline NLP $(n=4)$ & $4(100)$ \\
LP $(n=41)$ & $35(85)$ \\
Better than LP $(n=36)$ & $28(78)$ \\
\hline
\end{tabular}

Abbreviations: LP, light perception; NLP, no light perception; VA, visual acuity.

four cases. A remarkable difference between the two systems not noted with other pathogens.

Sensitivity testing was performed for all identified pathogens. All encountered pathogens were susceptible to our standard intravitreal antibiotic treatment with the combination of ceftazidime and vancomycin.

The chance of overall positive yield in relation to VA at presentation is depicted in Table 3. There was a trend for higher yield in worse presenting VA but this trend did not reach statistical significance $(P=0.438$; Pearson's $\chi^{2}$-test).

Vitreous samples were taken by tap in 41 cases and by vitrectomy in 44 cases. In the tap cases, $32(78 \%)$ showed overall growth, whereas positive growth was found in $37(84 \%)$ of vitrectomy cases. This difference was not statistically significant ( $P=0.476$; Pearson's $\chi^{2}$-test).

\section{Discussion}

Identifying the pathogen cause of endophthalmitis is a challenge. An eye cannot yield a large inoculation specimen, and obtaining it requires special care. In the presence of an inflamed eye with hazy anterior segment, collecting vitreous is a hazardous undertaking. The typical specimen size is therefore small. In addition, infections located in the anterior segment can lead to an intense anterior segment inflammatory response, whereas the microbial inoculum in the vitreous is limited. This reduces the likelihood of obtaining a positive culture from a vitreous specimen taken away from the site of infection. Organisms can also be slow growing as is typical of chronic endophthalmitis or have fastidious growth requirements as in the case of many endogenous endophthalmitis. For this reason, early studies have attempted to improve culture yields by using liquid broths. Analogous to vitreous, culturing of other normally sterile body fluids such as synovial, cerebrospinal, pleural, and peritoneal fluid is difficult. The use of BCB has been shown to improve yield in these fluids significantly. ${ }^{2}$

The reported yield of CM in endophthalmitis varies between 26 and $66 \% .^{5,6,8-11}$ Lower yields have been related to suboptimal access to laboratory facilities, ${ }^{6}$ and to infections due to other than postoperative endophthalmitis. ${ }^{10}$ Our yield in CM was $72 \%$, falling in the favorable end of the spectrum.

In contrast to earlier reports, we did not find $\mathrm{BCB}$ to be superior to CM. This could be due to a relatively good performance of $\mathrm{CM}$ in our institution. However, we did find that the combination of $\mathrm{BCB}$ and $\mathrm{CM}$ significantly improved the yield compared with $\mathrm{CM}$ alone. A combination of $\mathrm{CM}$ and broth medium was also used in the EVS, resulting in a high culture yield of $82 \% .^{12}$

A remarkable finding was a lower detection rate of the fastidious pathogen $H$. influenzae in BCB compared with $\mathrm{CM}$. Although numbers are low and therefore statistical grounds are missing, our results would suggest better performance of CM for this particular isolate. Concern about growth of $H$. influenzae in automated blood culture systems has been raised in earlier studies on the culture of normally sterile body fluids. Inadequate growth was reported for the Bactec system ${ }^{13}$ and later in the BacT/Alert FAN system $H$. influenzae and $H$. parainfluenzae were not identified in infected samples from normally sterile body fluids. ${ }^{14}$ This problem does not seem to occur in the culture of blood. Viability of $H$. influenzae depends on the presence of hemin (X-factor) and nicotine adenine dinucleotide (NAD, V-factor), ${ }^{14}$ normally present in blood. In the BacT/Alert FAN media, hemin is present but NAD is missing. ${ }^{14}$ Supplementing these factors, or adding blood to the BacT/Alert FAN 
system enables growth of $H$. influenzae. ${ }^{14}$ A reduction of viability due to lack of V-factor in our BCB media must therefore be assumed. In order to make the BacT/Alert FAN system more appropriate for the culture of ocular fluids, addition of NAD should be investigated further. Of our four cases of $H$. influenzae endophthalmitis, three cases followed phacoemulsification, whereas the remaining case was endogenous in origin. In the existing literature on endophthalmitis, this pathogen is rarely encountered. One large series of 250 consecutive cases of acute post-phacoemulsification endophthalmitis identified three cases in which $H$. influenzae was the causative pathogen. ${ }^{8}$ In a comprehensive review of 267 cases of endogenous endophthalmitis ${ }^{10}$ only one case was encountered. ${ }^{15}$ The fact that it is reported so infrequently as a causative pathogen in endophthalmitis likely reflects its fastidious growth characteristics. The risk of failure to detect $H$. influenzae should be considered when deciding to only use BCB systems for the work-up of endophthalmitis.

Vitrectomy allows a larger undiluted vitreous sample as compared with a tap. Use of a vitrectomy probe may also facilitate the sampling of formed vitreous without causing undue traction on the retina. It can also allow for a more directed sampling of vitreous in the area of known infection. However, despite these theoretical advantages, we did not find a significant difference in culture yield between the tap and the vitrectomy cases. A vitrectomy should therefore not be performed for the purpose of increasing the microbiological yield. This is in accordance with the recommendations of the EVS. ${ }^{11}$

Gram stains were performed in a subset of specimens. Although the sensitivity of the Gram stain was low, it had a very high positive-predictive value. These findings, again, are in agreement with the findings in the EVS. ${ }^{11}$ In the context of postoperative endophthalmitis, the outcome of the Gram stain will not have a direct impact on treatment. It could therefore be argued that the Gram stain can be omitted as a routine procedure in postoperative endophthalmitis. ${ }^{11}$ In endogenous cases, however, earlier recognition of the pathogen by Gram stain could guide the clinician in the choice of systemic antibiotics.

In conclusion, $\mathrm{BCB}$ is a good alternative to $\mathrm{CM}$ if access to microbiological facilities is suboptimal. We could not confirm superiority of BCB over CM, as claimed by earlier reports. In a setting with good access to both culture techniques, we showed that the combination of BCB and CM significantly increased the culture yield. As endophthalmitis is a devastating disease and targeted antimicrobial treatment is essential for preservation of visual function, optimization culture yields by the combined use of both systems should be considered.
Summary

What was known before

- Culture yield in bacterial endophthalmitis is suboptimal. Optimizing culture yield could improve treatment and outcome of endophthalmitis.

\section{What this study adds}

- Adding blood culture bottles to conventional media in the diagnosis of endophthalmitis improves culture yield from 72 to $81 \%$.

- Endophthalmitis is a devastating disease and targeted antimicrobial treatment is essential for the preservation of visual function. Optimizing culture yields by the combined use of both systems should be considered.

\section{Conflict of interest}

The authors declare no conflict of interest.

\section{References}

1 Ramakrishnan R, Bharathi MJ, Shivkumar C, Mittal S, Meenakshi R, Khadeer MA et al. Microbiological profile of culture-proven cases of exogenous and endogenous endophthalmitis: a 10-year retrospective study. Eye (Lond) 2009; 23(4): 945-956.

2 Simor AE, Scythes K, Meaney H, Louie M. Evaluation of the BacT/Alert microbial detection system with FAN aerobic and FAN anaerobic bottles for culturing normally sterile body fluids other than blood. Diagn Microbiol Infect Dis 2000; 37(1): 5-9.

3 von Essen R. Culture of joint specimens in bacterial arthritis. Impact of blood culture bottle utilization. Scand J Rheumatol 1997; 26(4): 293-300.

4 Eser I, Kapran Z, Altan T, Eren H, Yilmaz OF. The use of blood culture bottles in endophthalmitis. Retina 2007; 27(7): 971-973.

5 Joondeph BC, Flynn Jr HW, Miller D, Joondeph HC. A new culture method for infectious endophthalmitis. Arch Ophthalmol 1989; 107(9): 1334-1337.

6 Yospaiboon Y, Saree S, Pasadhika S. Blood culture and conventional media for vitreous culture in infectious endophthalmitis. J Med Assoc Thai 2005; 88(5): 639-642.

7 Kratz A, Levy J, Belfair N, Weinstein O, Klemperer I, Lifshitz T. Broth culture yield vs traditional approach in the work-up of endophthalmitis. Am J Ophthalmol 2006; 141(6): 1022-1026.

8 Pijl BJ, Theelen T, Tilanus MA, Rentenaar R, Crama N. Acute endophthalmitis after cataract surgery: 250 consecutive cases treated at a tertiary referral center in the Netherlands. Am J Ophthalmol 2010; 149(3): 482-487.

9 Recchia FM, Busbee BG, Pearlman RB, Carvalho-Recchia CA, Ho AC. Changing trends in the microbiologic aspects of postcataract endophthalmitis. Arch Ophthalmol 2005; 123(3): 341-346.

10 Jackson TL, Eykyn SJ, Graham EM, Stanford MR. Endogenous bacterial endophthalmitis: a 17-year prospective series and review of 267 reported cases. Surv Ophthalmol 2003; 48(4): 403-423.

11 Barza M, Pavan PR, Doft BH, Wisniewski SR, Wilson LA, Han DP et al. Evaluation of microbiological diagnostic 
techniques in postoperative endophthalmitis in the Endophthalmitis Vitrectomy Study. Arch Ophthalmol 1997; 115(9): 1142-1150.

12 Vine AK, Blodi BA, Elner SG, Johnson MW, Jessup LM, Pierson $\mathrm{CL}$ et al. Results of the Endophthalmitis Vitrectomy Study. A randomized trial of immediate vitrectomy and of intravenous antibiotics for the treatment of postoperative bacterial endophthalmitis. Arch Ophthalmol 1995; 113(12): 1479-1496.

13 Fuller DD, Davis TE, Kibsey PC, Rosmus L, Ayers LW, Ott $\mathrm{M}$ et al. Comparison of BACTEC Plus 26 and 27 media with and without fastidious organism supplement with conventional methods for culture of sterile body fluids. J Clin Microbiol 1994; 32(6): 1488-1491.

14 Pennekamp A, Zbinden R, von Graevenitz A. Detection of Haemophilus influenzae and Haemophilus parainfluenzae from body fluids in blood culture bottles. J Microbiol Meth 1996; 25: 303-307.

15 Stonecipher KG, Ainbinder DJ, Maxwell DP. Infectious endophthalmitis: a review of 100 cases. Ann Ophthalmol Glaucoma 1994; 26: 108-115. 